\title{
Exclusión renal por endometriosis profunda ureteral: a propósito de un caso
}

\section{Renal exclusion due to deep infiltrating ureteral endometriosis: a case report}

\author{
Tania Mergudich-Thal', Cristián Pomés-Correa², Ignacio San Francisco-Reyes ${ }^{3}$, \\ Milena Zamboni-Torres² y Claudia Celle-Traverso2* \\ ${ }^{1}$ Departamento de Obstetricia y Ginecología; ${ }^{2}$ Departamento de Ginecología; ${ }^{3}$ Departamento de Urología. Facultad de Medicina, Pontificia \\ Universidad Católica de Chile, Santiago, Chile
}

\section{Resumen}

Introducción: La endometriosis ureteral es una afección rara que afecta al 0.01-1,7\% de las mujeres con endometriosis. Hasta un $30 \%$ cursa de forma asintomática y un 11,5-14,7\% pueden evolucionar con falla renal. La falta de diagnóstico de la enfermedad puede terminar en una uropatía obstructiva y falla renal irreversible. Se presenta el caso de una paciente con afectación grave de la función renal secundaria a endometriosis profunda con compromiso ureteral. Caso clínico: Mujer de 35 años con endometriosis que consultó por exacerbación de los síntomas. En su estudio destaca, en la resonancia magnética, el hallazgo de endometriosis pélvica profunda y compromiso endometriósico intrínseco del uréter distal derecho, provocando una acentuada hidroureteronefrosis. El cintigrama renal demuestra acentuado compromiso de la función renal derecha, con una función relativa del 7\%. Se realizaron nefrectomía total derecha y resección de enfermedad pélvica profunda laparoscópica, sin incidentes. Conclusiones: La endometriosis ureteral representa un desafío diagnóstico y terapéutico. El manejo multidisciplinario entre radiólogos, ginecólogos y urólogos, mediante el diseño de una estrategia quirúrgica individualizada, es imprescindible para definir el tratamiento óptimo de estas pacientes.

Palabras clave: Endometriosis. Endometrioma. Obstrucción ureteral. Insuficiencia renal.

\section{Abstract}

Introduction: Ureteral endometriosis is a rare entity that affects $0.01-1,7 \%$ of women with endometriosis. Up to $30 \%$ of the patients are asymptomatic and $11.5-14.7 \%$ will develop renal failure. Misdiagnosis can lead to obstructive uropathy and permanent renal failure. We present the case of a patient with severe compromise of renal function secondary to deep infiltrating endometriosis with ureteral involvement. Case report: A 35-year-old woman with endometriosis presented with exacerbation of symptoms. Magnetic resonance showed deep pelvic endometriosis and intrinsic endometriotic involvement of the right distal ureter, causing a marked hydroureteronephrosis. Renal scintigram showed a severe compromise of the right renal function, with a relative function of $7 \%$. Through laparoscopy a total right nephrectomy and resection of deep infiltrating endometriosis was performed. The patient had a satisfactory recovery in the postoperative period. Conclusions: Ureteral endometriosis presents a diagnostic and therapeutical challenge. Joint multidisciplinary management between radiologists, gynecologists and urologists through the design of an individualized surgical strategy is essential to define the optimal treatment for these patients.

Key words: Endometriosis. Endometrioma. Ureteral obstruction. Renal failure.

$\begin{array}{ll}\text { Correspondencia: } & \text { Fecha de recepción: 13-03-2021 } \\ { }^{*} \text { Claudia Celle-Traverso } & \text { Fecha de aceptación: 21-10-2021 } \\ \text { E-mail: ccelle@med.puc.cl } & \text { DOl: 10.24875/RECHOG.M21000026 }\end{array}$

Disponible en internet: 23-12-2021 Rev Chil Obstet Ginecol. 2021;86(5):455-460 www. rechog.com 0048-766X / @ 2021 Sociedad Chilena de Obstetricia y Ginecología. Publicado por Permanyer. Éste es un artículo open access bajo la licencia CC BY-NC-ND (https://creativecommons.org/licenses/by-nc-nd/4.0/). 


\section{Introducción}

La endometriosis es una condición crónica que se define como la presencia de glándulas y estroma endometrial fuera de la cavidad endometrial ${ }^{1}$. Es una afección frecuente, que se presenta en el $5-15 \%$ de las mujeres en edad reproductiva y en el $3-5 \%$ de las mujeres posmenopáusicas. En orden decreciente, las ubicaciones más frecuentes son los ovarios $(54 \%)$, el ligamento ancho (35\%), el saco de Douglas (35\%) y los ligamentos uterosacros $(8 \%)^{2}$. Con menos frecuencia afecta el tabique rectovaginal, la región retrocervical y la vagina. Las localizaciones extragenitales 0 extrapélvicas son hallazgos poco frecuentes, aunque existen reportes de casos en prácticamente todos los órganos ${ }^{3}$.

La endometriosis se clasifica en tres tipos: peritoneal o superficial, ovárica y profunda. La endometriosis profunda consiste en la presencia de endometriosis subperitoneal, con invasión de al menos $5 \mathrm{~mm}$ de profundidad, e incluye lesiones en el espacio rectovaginal, los ligamentos uterosacros e infiltración de otros órganos abdominales y pélvicos, como el intestino, el colon rectosigmoide y el tracto urinario ${ }^{4,5}$. Un $14-20 \%$ de las pacientes con endometriosis presentan endometriosis profunda ${ }^{6}$.

La endometriosis del tracto urinario es una condición rara que afecta al $1-5,5 \%$ de las pacientes con endometriosis, y corresponde a la presencia de tejido endometrial sobre la vejiga, los uréteres, la uretra o los riñones ${ }^{6,7}$. Dentro de la endometriosis del tracto urinario, lo más frecuente es el compromiso vesical, en un $70-85 \%$, seguido por el ureteral en un $9-23 \%$, del riñón en un $4 \%$ y de la uretra en un $2 \%$.

El diagnóstico de endometriosis ureteral (EU) requiere una alta sospecha, ya que en la mayoría de los casos se presenta con síntomas inespecíficos o de forma asintomática hasta en un $30 \%$ de las pacientes ${ }^{3}$. Por ello, suele ser un hallazgo incidental durante la cirugía por enfermedad extensa. La EU afecta con más frecuencia el uréter distal y por lo general es unilateral (en el $83,82 \%$ de los casos), más comúnmente izquierda (en un $80 \%$, frente al $20 \%$ del lado derecho) ${ }^{6}$. Existen dos tipos: la EU extrínseca, cuando se encuentran glándulas endometriales y tejido estromal en la adventicia del uréter, y la EU intrínseca, cuando afecta al uroepitelio y la submucosa ${ }^{9}$. Esta última, menos frecuente en una relación 3:1, puede llevar a obstrucción ureteral, hidroureteronefrosis (HUN) y eventualmente pérdida de la función renal. Se ha descrito que el riesgo de compromiso de la función renal en los casos de EU es del 11,5-14,7\% ${ }^{10}$.

Se presenta el caso de una paciente con afectación grave de la función renal, asintomática, secundario a endometriosis profunda con compromiso ureteral. Debido a la baja frecuencia de la enfermedad, existe escasa evidencia sobre los algoritmos diagnósticos y las opciones de tratamiento. El objetivo de este trabajo es describir una forma silente y grave de presentación de EU y crear conciencia para favorecer un diagnóstico y un tratamiento precoces, y prevenir secuelas irreversibles en futuras pacientes.

\section{Caso clínico}

Mujer de 35 años, G2P1A1, con diagnóstico conocido de endometriosis (laparoscopia diagnóstica en 2014) y antecedente de resección de endometrioma ovárico por vía laparoscópica en 2017. Usó anticonceptivos orales entre 2014 y 2017, sin otro tratamiento hormonal posterior. Consultó con historia de dismenorrea intensa desde la menarquia que evolucionó a dolor pélvico constante invalidante posterior a la suspensión de los anticonceptivos orales, asociado además a dispareunia profunda, disquecia catamenial y disuria.

En la resonancia magnética (RM) de pelvis se constató endometriosis pélvica profunda extensa con compromiso del fondo de saco de Douglas y afectación bilateral de parametrios y de ligamentos uterosacros; además, compromiso endometriósico intrínseco del uréter distal derecho, provocando una acentuada HUN homolateral y afectación endometriósica mural de colon sigmoides distal, la unión rectosigmoidea y el polo cecal (Fig. 1).

Dada la HUN, se instaló un catéter doble $\mathrm{J}$ derecho y se realizó un cintigrama renal, que determinó un acentuado compromiso de la función renal derecha, con una función relativa del $7 \%$. Además, ante un aparente compromiso del colon, se completó el estudio con una colonoscopia que mostró solo compresión extrínseca del ciego y del colon sigmoides, sin compromiso de la mucosa.

Se realizó una intervención quirúrgica conjunta entre urología, ginecología y cirugía digestiva, por vía laparoscópica. El abordaje se llevó a cabo mediante cuatro puertos: uno de $10 \mathrm{~mm}$ supraumbilical para la óptica y tres accesorios de $5 \mathrm{~mm}$. En un primer tiempo quirúrgico se realizó una nefrectomía total derecha, seccionando el uréter homolateral proximal al punto estenosado. Luego se procedió a realizar el tiempo quirúrgico ginecológico, evidenciando el fondo de saco 


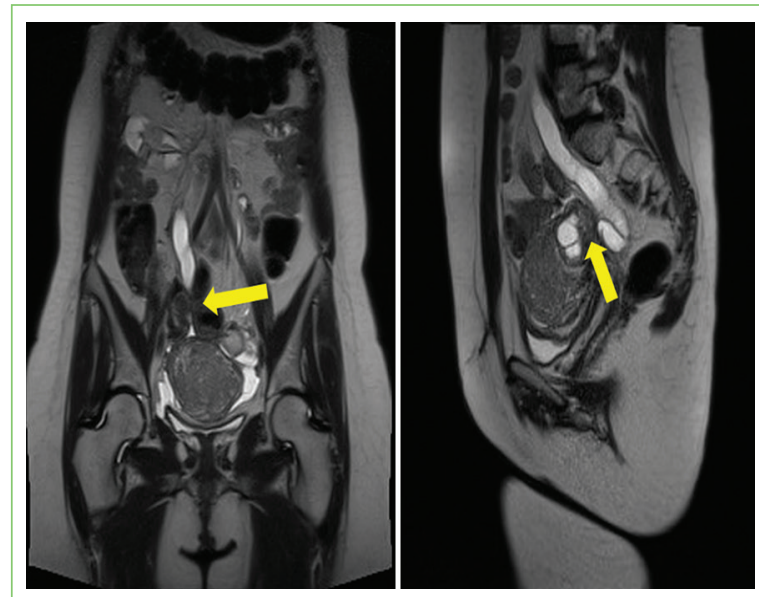

Figura 1. Resonancia magnética de abdomen y pelvis (T2). Se identifica el implante de endometriosis en el uréter distal derecho, con acentuada hidroureteronefrosis.

de Douglas sellado, el rectosigmoides y el ciego adherido a la cara posterior del útero y ambos ovarios. Una vez liberado el colon se identificó un nódulo endometriósico profundo dependiente del ligamento uterosacro derecho en íntimo contacto con la porción remanente del uréter y los vasos uterinos homolaterales. Previo a completar la histerectomía, se procedió a identificar el trayecto ureteral hasta identificar la región estenosante y el cruce con la arteria uterina, la cual se ligó con clips y se seccionó (Fig. 2). Se logró separar los vasos uterinos de inserción ureteral a vejiga, liberando el ligamento uterosacro. A continuación se completó la toma y sección de vasos uterinos y se logró finalmente completar la histerectomía total, extrayendo el útero, la trompa izquierda, el anexo derecho y el riñón derecho por la vagina. Finalmente se realizó una rectoscopia intraoperatoria para constatar la integridad de la mucosa colónica y descartar compresión extrínseca, con lo que se desestimó la necesidad de resección intestinal.

La paciente completó 3 días de posoperatorio y fue dada de alta en buenas condiciones, sin complicaciones.

El estudio anatomopatológico diferido de las piezas operatorias informó, para el riñón, atrofia hidronefrótica acentuada. En el segmento ureteral se observó la lámina propia edematosa, con numerosos focos de linfangiectasias y dilataciones vasculares, asociadas a infiltrado inflamatorio mixto con eosinófilos, focos

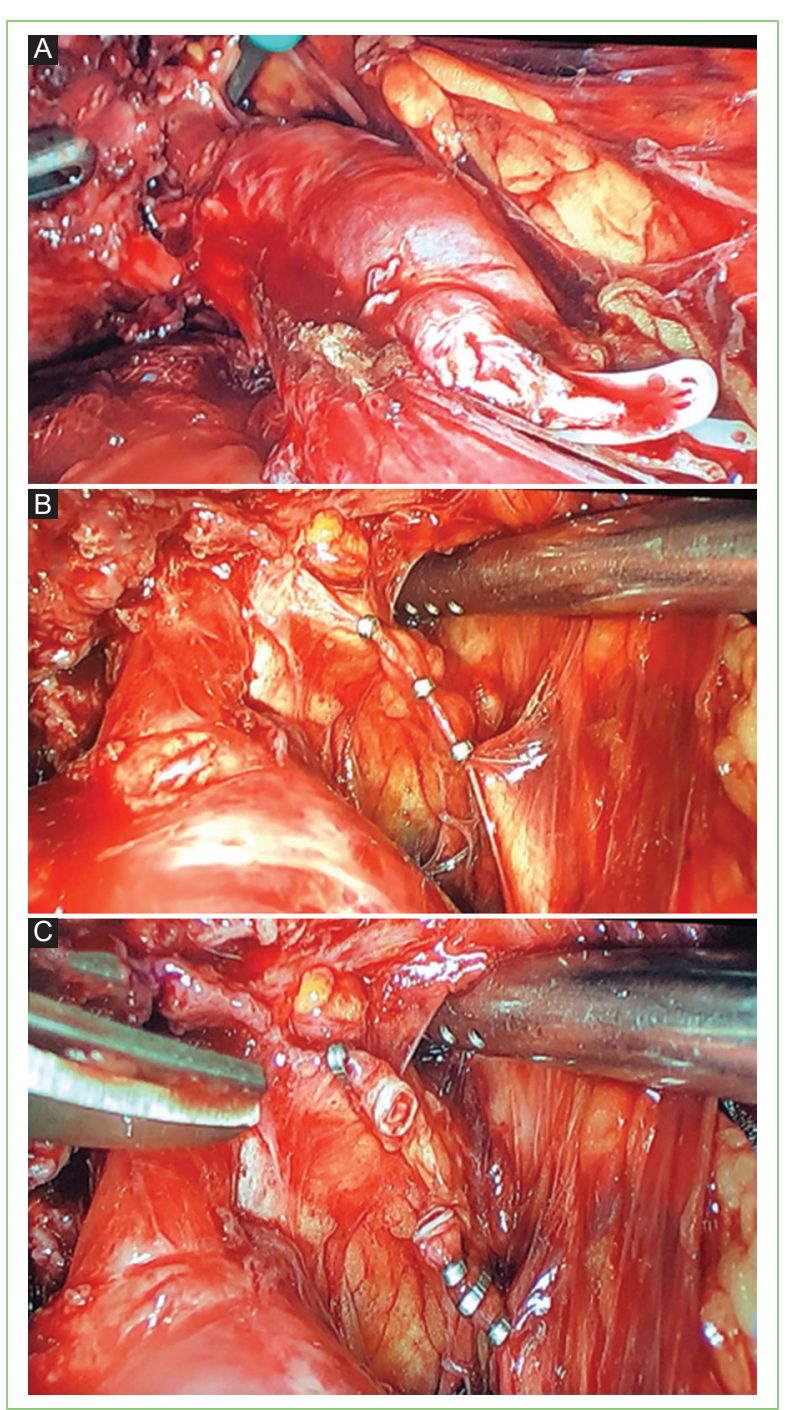

Figura 2. A: uréter derecho seccionado proximal a la zona estenosada. B: cruce con la arteria uterina ligada con clips. C: arteria seccionada.

granulantes y engrosamiento secundario mural. En la trompa uterina izquierda y en el cuerpo uterino también se observaron focos de linfangiectasias, y en este último adenomiosis e inflamación xantogranulomatosa con células gigantes asociadas a fibrosis hialina acentuada.

Llama la atención que en la biopsia de la pieza operatoria no se describe endometriosis ureteral. Esto se debe probablemente a que el uréter fue resecado por sobre el punto de estenosis.

La presencia de focos de linfangiectasias asociadas a infiltrado inflamatorio mixto con eosinófilos plantea como diagnóstico diferencial una hiperplasia angiolinfoide con eosinofilia. Sin embargo, la paciente 
no presenta ningún otro hallazgo en su historia, exploración física ni exámenes de laboratorio que permita plantear este diagnóstico de manera definitiva.

En el seguimiento hasta 1 año posoperatorio la paciente evoluciona favorablemente, con disminución significa del algia pélvica y la dispareunia, y mejoría de la calidad de vida. Se mantiene en tratamiento hormonal con dienogest. Se realiza seguimiento imagenológico con ecografía transvaginal que muestra un ovario remanente de aspecto sano. Además, en el seguimiento la función renal es normal y la ecografía renal muestra el riñón izquierdo de aspecto normal.

\section{Discusión}

La EU es una afección infrecuente, con una prevalencia que varía entre el $0,01 \%$ y el $1,7 \%$ en las pacientes con endometriosis según las distintas series reportadas en la literatura ${ }^{6}$. La prevalencia descrita es variable debido a la ausencia de sintomatología en gran parte de las pacientes y a la baja solicitud de exámenes imagenológicos sistemáticos para la evaluación de la vía urinaria en las pacientes con diagnóstico de endometriosis. El diagnóstico y el tratamiento oportuno de la EU son fundamentales, dado que en los casos graves puede llevar a serias complicaciones, como obstrucción ureteral silente y eventualmente falla renal $^{10}$. El compromiso de la función renal se ha reportado en un $11,5-14,7 \%$ de las pacientes con EU y en un $27 \%$ de aquellas con EU y HUN. Por ello, es importante tener una alta sospecha ${ }^{11}$.

La EU aislada es una forma de presentación poco frecuente. Por lo general, se asocia a endometriomas ováricos (52-68\% de los casos) u otras lesiones profundas, como implantes en el espacio rectovaginal $(47-56 \%$ de los casos), los ligamentos uterosacros (10-50\%) o el intestino (26-39\%)

La EU representa un desafío tanto en su diagnóstico como en su tratamiento. Igual que en el caso presentado, la mayoría de las pacientes no tienen síntomas urológicos específicos, sino síntomas inespecíficos asociados a la endometriosis, como dismenorrea, dispareunia o algia pélvica crónica. Aproximadamente el $30 \%$ de las pacientes con EU son por completo asintomáticas $^{3}$. Los síntomas urológicos específicos, como cólico renal cíclico o hematuria cíclica, son infrecuentes, presentándose en menos del $17 \%$ de las pacientes $^{5}$. Otras presentaciones mucho menos habituales reportadas en la literatura son anuria en pacientes monorrenas o hipertensión sin causa precisada ${ }^{6}$. Por esto, rara vez el diagnóstico de EU se realiza previo a la cirugía. El grado de sintomatología no se correlaciona con el grado de obstrucción ureteral'. La exploración física suele ser normal, pero la palpación de un nódulo en el fondo de saco de Douglas o en los ligamentos uterosacros debe aumentar la sospecha de EU ${ }^{12}$.

Según un análisis multivariado realizado por $\mathrm{Hu}$ et al. ${ }^{10}$, presentar endometriosis en estadio IV de la clasificación revisada de la Sociedad Americana de Fertilidad, lesiones endometriósicas del ligamento uterosacro $\geq 3 \mathrm{~cm}$ y el antecedente de cirugía por endometriosis son factores de riesgo independientes para EU en pacientes con endometriosis profunda. Otros estudios también demostraron un índice de masa corporal significativamente más bajo en las pacientes con endometriosis profunda con compromiso ureteral en comparación con las pacientes sin compromiso ureteral. Por lo tanto, en las pacientes que presenten alguno de estos factores de riesgo la sospecha de EU debe ser mayor ${ }^{13}$.

El método de referencia para el diagnóstico de la EU es la laparoscopia exploradora, que tiene un fin tanto diagnóstico como terapéutico. Sin embargo, tener el diagnóstico preoperatorio permite una mejor planificación quirúrgica y así disminuir los riesgos asociados.

Si bien existen numerosos estudios radiológicos que permiten sospechar y confirmar la EU, actualmente no existe un consenso unánime sobre qué técnica diagnóstica se debe utilizar ${ }^{6}$.

La ecografía abdominal es un examen simple, no invasivo, y los hallazgos son altamente sensibles para la HUN. Debido a la alta tasa de presentación asintomática de la EU, se recomienda su uso sistemático para descartar una obstrucción del tracto urinario en pacientes con diagnóstico de endometriosis profunda, sobre todo cuando los nódulos son de tamaño $\geq 3$ $\mathrm{cm}^{10}$. En la práctica clínica, en general se sugiere solicitar una ecografía abdominal periódica (cada 6 meses) desde el momento del diagnóstico ${ }^{14}$.

En las mujeres con HUN se deben solicitar estudios radiológicos adicionales, como tomografía computada (TC) o RM, para identificar de mejor manera las lesiones endometriósicas y los sitios de estenosis ${ }^{3}$. Si bien la TC es el mejor estudio imagenológico para la evaluación de los riñones, los uréteres y la vejiga, y puede ayudar a identificar la relación entre las lesiones y estas estructuras, muchos autores consideran la RM como la prueba de elección ante la sospecha de EU. La RM permite visualizar todos los componentes del sistema urinario, explorar todas las ubicaciones pélvicas de la endometriosis y diferenciar lesiones de 
otro origen, como neoplasias ${ }^{5}$. La RM revela signos directos de endometriosis ureteral, como un nódulo que invade el uréter a lo largo de su trayecto o en la unión ureterovesical, y es capaz de diferenciar compromiso extrínseco e intrínseco (aunque suele sobreestimar el intrínseco). Las secuencias más útiles para la evaluación del tracto urinario son las potenciadas en $\mathrm{T} 2$ en el plano coronal ${ }^{10}$. La RM tiene una mayor sensibilidad (91\% frente a $82 \%)$, pero menor especificidad (59\% frente a $67 \%$ ), que la cirugía para el diagnóstico de EU intrínseca ${ }^{14}$.

Otras técnicas, como la ureteropielografía retrógrada - la pielografía endovenosa, han sido tradicionalmente utilizadas para evaluar a pacientes con sospecha de endometriosis del tracto urinario, pero en la actualidad han sido reemplazadas por la $\mathrm{RM}^{3,6}$.

En las pacientes con estenosis ureteral grave se debe evaluar la función residual del riñón afectado con un cintigrama renal o renograma. Cuando la función renal es $<14 \%$ existe un mayor riesgo de pielonefritis y de hipertensión renovascular, por lo que clásicamente se recomienda la nefrectomía ${ }^{11}$.

El manejo de las pacientes con endometriosis ureteral debe ser con un equipo multidisciplinario que incluya radiólogos, ginecólogos y urólogos. El objetivo del tratamiento es aliviar la obstrucción, eliminar los síntomas y preservar la función renal. La elección del tratamiento dependerá de la edad de la paciente, el deseo de fertilidad, la extensión de la enfermedad, la gravedad de los síntomas, el grado de alteración de la función renal, la gravedad de la obstrucción y el nivel del uréter afectado. Dentro de las opciones terapéuticas se encuentran la terapia hormonal y la cirugía; sin embargo, no hay evidencia contundente que las compare.

La terapia hormonal está indicada para el manejo sintomático del dolor en pacientes con endometriosis profunda sin obstrucción ureteral. Como primera línea se recomiendan los anticonceptivos hormonales, por su efectividad y seguridad en el uso a largo plazo ${ }^{5}$. Los análogos de la hormona liberadora de gonadotropina se recomiendan como segunda línea, dados sus efectos adversos producidos por el hipoestrogenismo ${ }^{6}$. La terapia hormonal está contraindicada como primera línea en pacientes con obstrucción ureteral, ya que, si bien puede disminuir el tamaño de las lesiones, no actúa sobre el tejido fibrótico y adherencial, que podría progresar de manera silente llevando a falla renal. El papel de la terapia hormonal en las pacientes con EU sin obstrucción ureteral es poco claro; se podría utilizar para el manejo sintomático en caso de dolor o mientras se planifica una cirugía ${ }^{10}$.

La cirugía está indicada en las pacientes con EU sintomáticas o con algún grado de HUN. El abordaje quirúrgico suele ser complejo por la dificultad intrínseca del tratamiento de la endometriosis y por las frecuentes cirugías previas que dan lugar a adherencias y distorsión anatómica. Esto requiere la participación de cirujanos expertos en laparoscopia, con amplia experiencia en esta enfermedad ${ }^{11}$.

Dentro de los procedimientos actualmente disponibles se encuentran la ureterólisis, la ureteroneocistostomía, la resección ureteral parcial con anastomosis primaria y otras formas de reconstrucción. El tratamiento quirúrgico puede realizarse mediante técnicas mínimamente invasivas o por laparotomía. La cirugía mínimamente invasiva permite una visión magnificada de la pelvis, proporciona una mejor exposición y se asocia a menor dolor posoperatorio, menos días de hospitalización y mejor convalecencia ${ }^{8}$.

El enfrentamiento quirúrgico de la endometriosis ureteral aún es controversial, dado que no existe evidencia de buena calidad que compare los resultados de distintas intervenciones, principalmente debido a la baja frecuencia de la enfermedad y a la dificultad para realizar ensayos controlados aleatorizados. La mayoría de los estudios disponibles son retrospectivos, no controlados, e incluyen poblaciones muy heterogéneas.

Al revisar la literatura, en general, en los casos de endometriosis extrínseca, cuando la afectación ureteral es leve, se prefiere realizar ureterólisis con extirpación de los focos endometriósicos. Cuando esto no es suficiente para liberar el uréter y restablecer su anatomía y su función normal, como en los casos de afectación ureteral moderada a grave (HUN moderada o grave), en especial con afectación intrínseca del uréter, se prefiere la resección del segmento ureteral afectado con ureteroneocistostomía o anastomosis uretero-ureteral primaria (dependiendo de la ubicación de los implantes endometriósicos y de la longitud del uréter remanente) $)^{7,8,10}$. Sin embargo, estudios más recientes han demostrado resultados satisfactorios con ureterólisis laparoscópica conservadora incluso en pacientes con EU y HUN moderada/grave, con una recurrencia del $3,9 \%$. En casos de endometriosis extensa, la cirugía generalmente incluye histerectomía con salpingectomía bilateral (en pacientes que no desean mantener su fertilidad) y nefrectomía (frente a exclusión renal) ${ }^{14-16}$.

En el estudio de Hu et al..$^{10}$, a tres pacientes con HUN y compromiso grave de la función renal no se les realizó nefrectomía argumentando que esta conlleva un 
aumento de las complicaciones durante y después de la cirugía, y un mayor requerimiento de transfusiones. Se describe que ninguna paciente desarrolló infecciones ni dolor persistente durante los 2 años de seguimiento. Sin embargo, esta no es la práctica habitual.

\section{Conclusiones}

La EU es una afección rara e infrecuente, que en casos graves puede llevar a serias complicaciones incluso en ausencia de síntomas urológicos específicos. Por lo tanto, es fundamental tener una alta sospecha en todas las pacientes con diagnóstico de endometriosis profunda y buscarla dirigidamente con ecografías abdominales periódicas. Ante una alta sospecha, la RM y el cintigrama renal pueden ayudar a confirmar la EU y a establecer la función renal previamente a la cirugía. El manejo multidisciplinario entre radiólogos, ginecólogos y urólogos, mediante el diseño de una estrategia quirúrgica individualizada, es imprescindible para definir el tratamiento óptimo de estas pacientes. Por último, son necesarios estudios controlados que comparen los resultados de las distintas intervenciones quirúrgicas y contribuyan a decidir el manejo óptimo en cada paciente.

\section{Financiamiento}

No hubo fuentes de financiamiento.

\section{Conflicto de intereses}

Este trabajo no cuenta con conflictos de intereses.

\section{Responsabilidades éticas}

Protección de personas y animales. Los autores declaran que para esta investigación no se han realizado experimentos en seres humanos ni en animales.
Confidencialidad de los datos. Los autores declaran que han seguido los protocolos de su centro de trabajo sobre la publicación de datos de pacientes.

Derecho a la privacidad y consentimiento informado. Los autores han obtenido el consentimiento informado de los pacientes y/o sujetos referidos en el artículo. Este documento obra en poder del autor de correspondencia.

\section{Bibliografía}

1. Torres Gómez F, Vázquez Ramírez F, Torres Olivera F. Endometriosis ureteral. A propósito de un caso. Arch Esp Urol. 2006;59:192-5.

2. Martínez-Varea A, Vila-Vives J, Molina-Planta M, López-Acón J, Ruiz-Cerdá J, Payá-Amate $\mathrm{V}$, et al. Manejo quirúrgico de la endometriosis vésico-ureteral y sus complicaciones. Rev Chil Obstet Ginecol. 2012;77:397-400.

3. Bernal J, Tapia V, Astroza G. Endometriosis del tracto urinario: revisión de la literatura. Revista Chil Urol. 2014;79:54-8.

4. Freire MJ, Dinis PJ, Medeiros R, Sousa L, Aguas F, Figueiredo A. Deep infiltrating endometriosis - urinary tract involvement and predictive factors for major surgery. Urology. 2017;108:65-70.

5. Palla VV, Karaolanis G, Katafigiotis I, Anastasiou I. Ureteral endometriosis: a systematic literature review. Indian J Urol. 2017;33:276-82.

6. Barra F, Scala C, Biscaldi E, Vellone V, Ceccaroni M, Terrone C, et al. Ureteral endometriosis: a systematic review of epidemiology, pathogenesis, diagnosis, treatment, risk of malignant transformation and fertility. Hum Reprod Update. 2018;24:710-30

7. Knabben L, Imboden S, Fellmann B, Nirgianakis K, Kuhn A, Mueller M. Urinary tract endometriosis in patients with deep infiltrating endometriosis: prevalence, symptoms, management, and proposal for a new clinical classification. Fertil Steril. 2015;103:147-52.

8. Talreja D, Salunke V, Pande S, Gupta C. Successful management of ureteric endometriosis by laparoscopic ureterolysis - a review and report of three further cases. Arab J Urol. 2018;16:342-9.

9. Jadoul P, Feyaerts A, Squifflet J, Donnez J. Combined laparoscopic and vaginal approach for nephrectomy, ureterectomy, and removal of a large rectovaginal endometriotic nodule causing loss of renal function. J Minim Invasive Gynecol. 2007;14:256-9.

10. Hu Z, Li P, Liu Q, Zhu H, Sun $Y$, Zhao X, et al. Ureteral endometriosis in patients with deep infiltrating endometriosis: characteristics and management from a single-center retrospective study. Arch Gynecol Obstet. 2019;300:967-73.

11. Ota K, Sato K, Tanaka M. Ureteral stenosis due to DIE (deep infiltrating endometriosis) with difficulty in treatment: case report and brief literature review. Gynecol Minim Invasive Ther. 2017;6:214-6.

12. Mu D, Li X, Zhou G, Guo H. Diagnosis and treatment of ureteral endometriosis: study of 23 cases. Urol J. 2014;11:1806-12.

13. Raimondo D, Mabrouk M, Zannoni L, Arena A, Zanello M, Benfenati A, et al. Severe ureteral endometriosis: frequency and risk factors. J Obstet Gynaecol. 2017;38:257-60.

14. Maccagnano C, Pellucchi F, Rocchini L, Ghezzi M, Scattoni V, Montorsi $F$, et al. Ureteral endometriosis: proposal for a diagnostic and therapeutic algorithm with a review of the literature. Urol Int. 2013:91:1-9.

15. Mason R, Alamri A, Gusenbauer K, Kapoor A. Intrinsic ureteral endometriosis as a cause of unilateral obstructive uropathy. Can Urol Assoc J. 2016;10:E119-21.

16. Cavaco-Gomes J, Martinho M, Gilabert-Aguilar J, Gilabert-Estélles J. Laparoscopic management of ureteral endometriosis: a systematic review. Eur J Obstet Gynecol Reprod Biol. 2017;210:94-101. 